\title{
SOME CONSIDERATIONS ASSOCIATED WITH HIGH CURRENT ACCELERATOR ION SOURCES*
}

\author{
Th.J.M. Sluyters \\ Brookhaven National Laboratory \\ Upton, New York
}

During the past decade the goal of most machine bullders has changed considerably. Initial$1 y$, energy was most important. Later, when the beam intensity became the center of interest, machine builders realized the importance of high quality beams. In the early days of most linear accelerators, milliamperes of beam current was considered large; at present, more than a hundred milliamperes is being accelerated in the same machine but its quality reduces the effective use of all of it.

Improvements of preaccelerators and low energy transport systems were begun in order to minimize deterioration of the beam quality before injection. Preacceleration and transport systems seem to be well understood. Accelerating tubes and transport elements can now be built which do not significantly distort the emittance. 1,2

Now much of the problem of less performance can be attributed to nonideal extraction of beams from ion sources. A beam of good optical quality has to be injected into preaccelerators, that means, injection of a beam as uniform as possible and with an emittance as small as possible. The difference between ideal and nonideal extraction from an ion source is best demonstrated by comparing fluorescent beam images after extraction obtained after a slotted plate on a quartz flag. Figure 1 shows split images from individual slots. The multiple images may be explained by a distortion of the ion emitter. It is evident that the emittance area is reduced considerably if the split images can be squeezed together. This distortion can be considered as the most serious aberration. Another type of aberration is the spherical aberration, as shown in Fig. 2. Ideal beam extraction shows single images as demonstrated in Fig. 3.

The control of the shape of the plasma boundary is the main problem of the builder of each high intensity accelerator ion source and more specifically the duoplasmatron.

\section{Standard or Three-Electrode Duoplasmatron Ion Source}

The duoplasmatron is the standard source for most accelerators. It has proven to be reliable in continuous and pulsed operation over a wide range of beam currents. Some recently constructed very high intensity duoplasmatrons are now operational at:

\footnotetext{
* Work performed under the auspices of the
}

U.S. Atomic Energy Commission. a) Saclay, with $100 \mathrm{~mA}$ beam current and a norma1ized apparent emittance ${ }^{t}$ of $\sim 0.04 \mathrm{~cm}$-rarad. ${ }^{3}$

b) Brookhaven, with $150 \mathrm{~mA}$ and an emittance of $0.16 \mathrm{~cm}$-mrad. 4

c) Serpukhov (USSR), with $300 \mathrm{~mA}$ and an enittance of $0.32 \mathrm{~cm}-\mathrm{mrad} .5$

d) CERN, with $500 \mathrm{~mA}$ and an emittance of $0.6 \mathrm{~cm}-\mathrm{mrad} .6$

Figure 4 shows the principle of the threeelectrode duoplasmatron. Its operation can be summarized as follows: the plasma closest to the anode is formed by the electrons from the cathode plasma, channeled by the geometry of the intermediate electrode (I.E.) and by the axial nonuniform magnetic field between I.E. and anode. The consequently high electron intensities cause strong ionization of the gas creating considerable axial density gradients in front of the anode, producing locally a positive potential ( $20 \mathrm{eV})$ with respect to the anode. Positive ions are, therefore, ejected through the anode aperture together with those electrons that can pass the potential barrier between the dense plasma and the anode.7, It now depends on the anode geometry downstream of the aperture and the extraction potential where the plasma boundary is located.

\section{Extraction Systems}

There are basically two extraction methods:

1) Extraction from a smal1, high density discharge area inside the aperture of the anode, as is shown in Fig. 5. This method, which was applied initially in almost all machines, leads to large divergences in the beam. However they can produce very clean beams.

2) Extraction from an expanded plasma. The ions and electrons are ejected through the anode aperture into a cylindrical cup that shields the plasma from external fields (see Fig. 4). In this way the local ion density may be reduced to levels where relatively low extraction voltages can be applied. The required high currents with small beam divergence is convenient for the design of the beam optics of the preaccelerator.

${ }^{\dagger}$ Definition of normalized emittance $E$ of a beam with cylindrical symmetry is the smallest ellipse surrounding all particles in a two-dimensional phase-plane area $r-r^{\prime}$ divided by $T$ and multiplied by $\beta Y . \quad \beta=v / c$ and $Y=\left(1-\beta^{2}\right)^{-\frac{1}{2}}$. Such a definition allows comparison of emittances independent of energy. 
As mentioned above, a dense beam of ions and electrons moves through the anode hole into the cup. The beam optical system starts at the ion emitter, which is assumed to be located at the exit edge of the expansion cup. This is a convenient assumption for calculations of Pierce extraction. The emitter adjusts itself such that there is equilibrium between space-charge forces, and external magnetic and electric fields. The ideal shape for the ion emitter (see Fig. 4) is a surface without protrusions that can only be obtained with a uniform ion density distribution at the surface. From emittance measurements one may infer shapes such as shown in Fig. 6. If the anode aperture is small ( $\sim 1 \mathrm{~mm}$ ) compared with the diameter of the discharge column $(\sim 4 \mathrm{~mm})$ we can assume uniform transverse density; the number of extracted ions is then proportional with the aperture area. When the expansion cup is magnetically shielded by a "septum" from the discharge side of the anode and the cup length is kept short, a minimum of beam disturbance and beam loss occurs during expansion. We call this "close-coupled" extraction. These principles are applied in the operational Brookhaven source shown in Fig. 7; its characteristics are summarized in Table I. It should be noted that the stray magnetic field inside our cup is very small due to the septum and that this field is zero $3 \mathrm{~mm}$ downstream from the anode aperture at the operational magnet current.

Another example of "close-coupled" extraction is demonstrated in an experiment at the Rutherford Laboratory with a very small expansion cup $(\phi=0.060 \mathrm{in}$.$) that approximates extraction from$ the discharge area itself. 9 In both examples the ion emitter has no measurable bumps, as demonstrated by emittance measurements.

The control of the ion emitter for larger expansion cups is more difficult. Several physical phenomena $c a n$ disturb the shape of the plasma boundary:

a) Stray fields in the cup, particularly when a "mild steel" septum cannot be applied due to temperature problems. In such cases one replaces the septum by molybdenum or tungsten buttons. By lengthening the expansion cups, the plasma boundary can be protected from stray field, but that increases ion losses on the walls. Small compensation coils around the expansion cup are sometimes employed to reduce radial diffusion. 6,8 The advantage of additional magnetic fields for increasing the beam current remains disputable. In most applications it disturbs the boundary despite much care in their construction and can easily cause "hollow" beams.10 To my knowledge, magnetic field control by conical cup shapes has been explored only for lower densities 11 (see Fig. 8).

b) The "plasma" in the cup is not neutral; there is an electron excess, originating in the discharge plasma. The mean energies of these electrons seem to correspond roughly with the intermediate electrode potential. These electrons influence the ion density distribution, which keeps the center of the plasma denser. They also ionize the rest gas forming a second (isotropic) plasma. Some control of the electron and/or ion density by isolating or biasing the cup walls has been reported both by Orsay and Saclay. 8,12 The reliability of this technique is stil1 under investigation.13

In many applications one is inclined to establish a parallel uniform beam at the extractor aperture. In those cases, Pierce geometry of the electrodes in the extractor region is normally chosen. The axial potential distribution $v_{z}=$ const $z^{4 / 3}$ has to be established across the diameter of the beam by properly shaping the electrodes. For a current density of $100 \mathrm{~mA} / \mathrm{cm}^{2}$ the constant is $1.5 \times 10^{4}$. The location of the ion emitter can be more or less fixed at the end of the cup by a small ridge in the cup exit without significant reduction of intensity. The Pierce shape of the anode close to the ion emitter appears in practice not too critical.

\section{Theoretical and Experimental Emittance Values}

Based on the theory of a directed plasma (neglecting the contribution of the isotropic cup plasma) ejected through the anode aperture, the emittance value may be estimated from the cup geometry and the ion velocity. The velocity can be derived from the energy with which the ions are ejected into the cup. An ion energy spectrum in the cup measured with different methods 8 shows values for $k T_{i}$ between 10 and $100 \mathrm{eV}$, so that for $20 \mathrm{eV}$ $v_{\text {ion }}=\sqrt{2 \mathrm{kT}_{\mathbf{i}} / \mathrm{m}}=9 \times 10^{4} \mathrm{~m} / \mathrm{sec}$. The normalized emittance is now:

$$
E=\frac{(\text { two-dimensional phase-space area) }}{\pi} \beta Y
$$

and, for $\gamma=1$,

$$
E=\frac{v}{c} \frac{\text { area }}{\pi}=3 \times 10^{-4} \frac{\text { area }}{\pi} \mathrm{cm}-\mathrm{mrad} .
$$

An experimental verification of the directed plasma theory should be: reduction of the emittance by a factor of two when the cup length is increased. by two. Figure 9 compares emittances obtained from two expansion cups which differ only in their cup lengths. The expansion cups are magnetically well shielded in both examples. The insert serves to protect the cylinder from backstreaming electrons originating in the accelerating column. The best experimental agreement and the smallest emittance $(0.007 \mathrm{~cm}-\mathrm{mrad})$ have been obtained by Wroe ${ }^{9}$ with an expansion cup of very small dimension. 1 These results fit the theory of "close coupling" well.

Emittances tend to change as a function of time. Reducing the pulse length reduces as well the emittance. For instance, for the Brookhaven source, if the pulse length is decreased from $120 \mathrm{usec}$ to $40 \mathrm{usec}$, the total emittance is reduced by about $30 \% .4$ We can, therefore, expect that the instantaneous emittances are much smaller than the integrated measurements suggest. This might only be caused by a fluctuating ion emitter.

The ion emitting surface can be fixed and shaped by a highly transparent coarse grid at the exit of the plasma cup.13 For high intensity beams the destruction of such grids by backstreaming electrons is still a problem. 
It appears that the apparent emittance increases with beam current. This can be explained by the higher anode voltage required for higher currents. It should increase the maximum energies with which the protons enter the cup and therefore the emittance.

\section{Modified or Four-Electrode Duoplasmatron}

Although with the classic three-electrode duoplasmatron high beam currents can be produced, it remains difficult to obtain currents in excess of $100 \mathrm{~mA}$ with uniform density distribution and distortion-free plasme boundary.

There is some evidence that the ion density in the radial direction of the discharge column in a Penning source is rather uniform. ${ }^{14} \mathrm{~A}$ modified duoplasmatron incorporating the Penning source idea seems to bę an encouraging development.

This type of duoplasmatron was first reported in 1963 by Demirkhanov. 15 The conventional duoplasmatron configuration is extended with a mild steel reflecting electrode (R.E.) and the steel anode is replaced by a copper (nonmagnetic) one (Fig. 10). The electrons from the cathode plasma injected into an extended anode region oscillate between the I.E. and R.E.; these electrodes are both negative with respect to the anode and bridged by a magnetic field. The large distance traveled by electrons enhances the ionizing of the gas, allowing lower gas pressure $\left(\sim 10^{-2} \mathrm{mmHg}\right)$. Therefore, the aperture in the R.E. can be larger than for the standard source. The effect of stray magnetic field in the cup can be reduced by the cone shaped as discussed before.

Two recent publications reflect the advantages of this type of ion source for high beam intensities. Ulbricht reports "uniform" beam density for beam currents around $200 \mathrm{~mA} .16$ A more recent result from Fasolo demonstrates that beam currents of $300 \mathrm{~mA}$ can be obtained with normalized emittances as 1 ow as $0.08 \mathrm{~cm}-m r a d .10$ In both publications the basic source dimensions of Demirkhanov, who obtained emission currents more than $1 \mathrm{~A}$, have been app1 ied.

\section{Conclusion}

The necessity of good optical beam quality for large intensity beams has stimulated a more systematic research in the formation of the plasma boundary in large expansion cups.

It seems to be preferable to extract very high intensity beam currents from properly shielded short expansion cups ("close-coupled" extraction).
The modified duoplasmatron with four electrodes might become more attractive for very high beam current production than the classic duoplasmatron.

The adjustments of individual sources for optimüm performance is still vcry delicate and requires lengthy experimental programs. Discussed studies, however, show promising beam quality results for future very intense machines.

\section{References}

1. Th.J.M. Sluyters, Proc. Proton Linear Accelerator Conference, Brookhaven, 1968, BNL 50120 (C-54), p. 228 .

2. C. Agritellis, R. Chasman, and Th.J.M. Sluyters, Proceedings of this Conference.

3. J. Faure, Proc. Proton Linear Accelerator Conference, Brookhaven, 1968, BNL 50120 (C-54), p. 240 .

4. Th.J.M. Sluyters et al., Proc. VI Intern. Conf. High Energy Accelerators, Cambridge, Mass., 1967, P. 275 .

5. V.A. Batalin et al., ibid., P. Al.

6. B. Vosicki et a1., Proc. 1966 Linear Accelerator Conference, Los Alamos, LA-3609, p. 344.

7. M.D. Gabovich, AD 623-822, Translation, 1965.

8. G. Gautherin, Thesis, Orsay, 1967.

9. H. Wroe, Nuc1. Instr. \& Methods 58, 213 (1968).

10. J.A. Fasolo, Proc. Proton Linear Accelerator Conference, Brookhaven, 1968, BNL 50120 (C-54), p. 306 .

11. N.B. Brooks et a1., Rev. Sci. Instr. 35, 894 (1964).

12. P. Bernard et al, Proc. VI Intern. Conf. High Energy Accelerators, Cambridge, Mass., 1967, p. A8.

13. 0. Reifenschweiler et al., Nuc1. Instr. \& Methods $\underline{30}, 298$ (1964).

14. E. Heiniche et a1., Nuc1. Instr. \& Methods 58, 125 (1968).

15. R.A. Demirkhanov et a1., High Energy Papers from USSR, BNL 767 (C-36), 1963, p. 224.

16. P. Ulbricht, Institut fïr Plasmaphysik, Garching, Munchen, 1967. 
TABLE I

Characteristics of the Operational Brookhaven Source

$\begin{array}{ll}\text { Filament current } & 30 \mathrm{~A} \\ \text { Anode voltage } & 250 \mathrm{~V} \\ \text { Magnet } & 750 \mathrm{~A} \times \text { turns } \\ \text { Maximum B field } & \sim 1 \mathrm{kG} \\ \text { B field at cup exit } & 0 \mathrm{G} \\ \text { Cup size } & \text { Width } 12 \mathrm{~mm}, \text { length } 10 \mathrm{~mm} \\ \text { Gap I.E. - anode } & 8 \mathrm{~mm} \\ \text { Anode aperture } & 1.2 \mathrm{~mm} \\ \text { Extractor-cup distance } & 16.5 \mathrm{~mm} \\ \text { Anode material } & \text { Mild steel septum with tungsten } \\ \text { Discharge current } & \text { insert (Fig. 7) } \\ \text { Pulse shape } & 40 \mathrm{~A} \\ \text { Pulse length } & -7 \\ \text { Beam current } & 130 \mathrm{msec} \\ \text { Normalized phase-space area } & 150 \mathrm{~mA} \\ \end{array}$

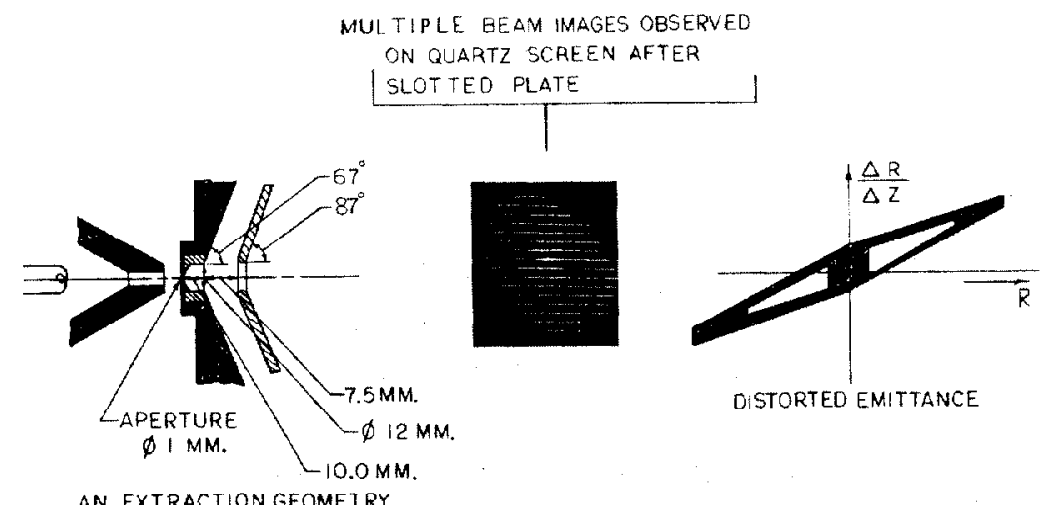

AN EXTRACTIONGEOMETRY

Fig. 1. Example of Non-Ideal Extnaction.

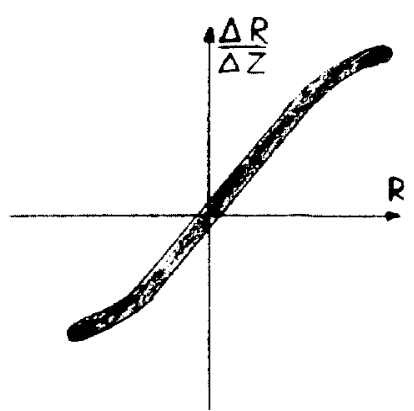

Figure 2. 

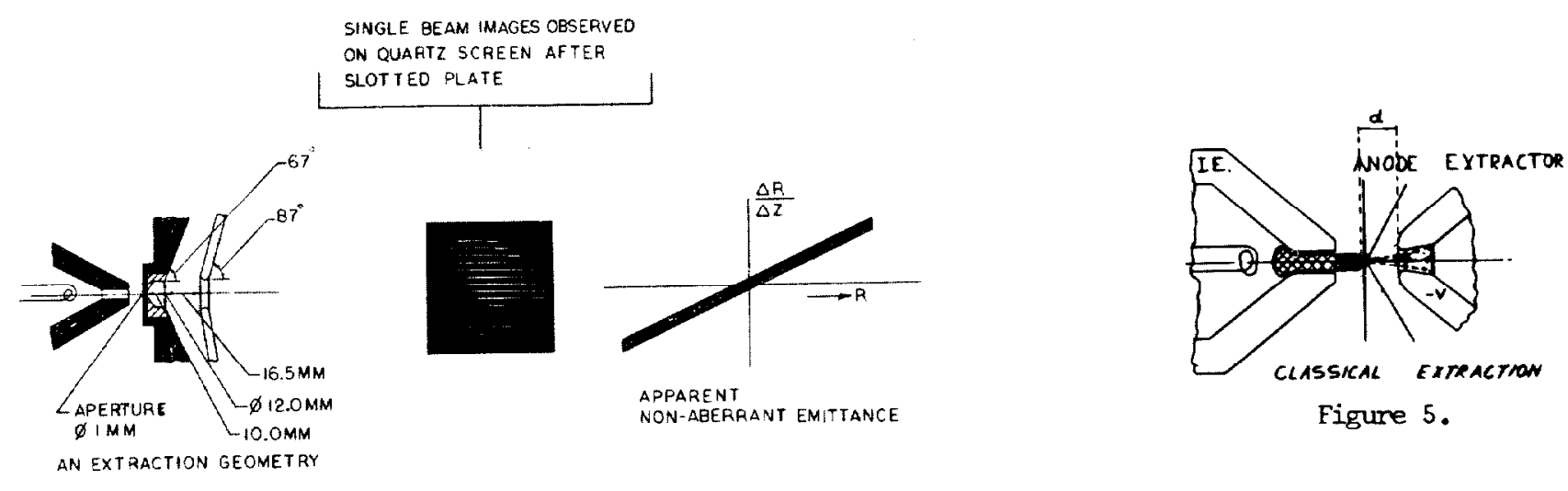

Figure 5.

Fig. 3. Example of Ideal Extration.
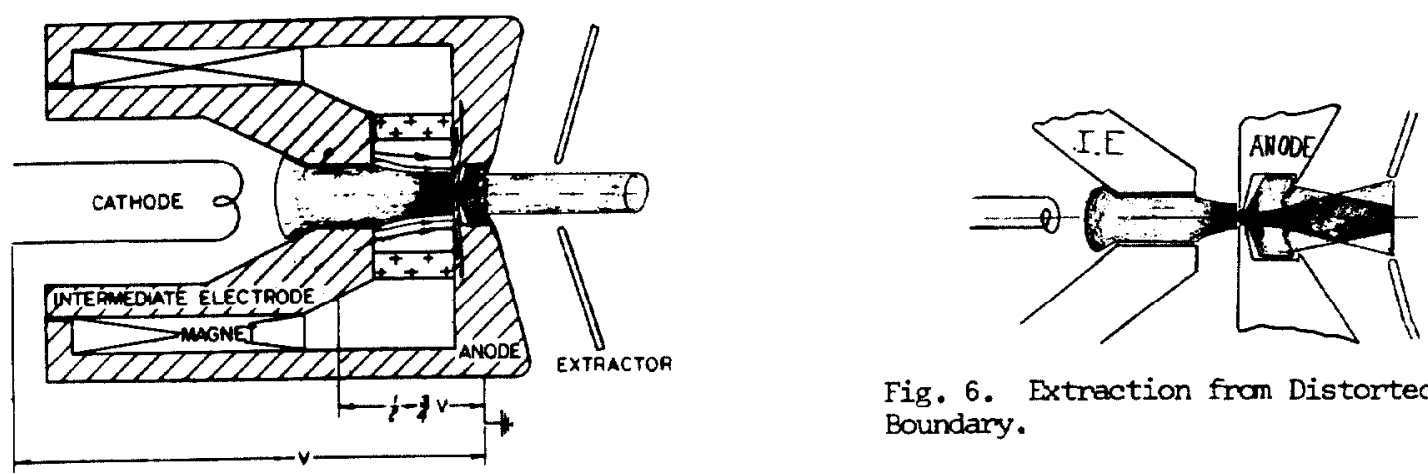

Fig. 6. Extraction from Distorted Plasma Boundary.

Fig. 4. Three Electrode Duoplasmatron.

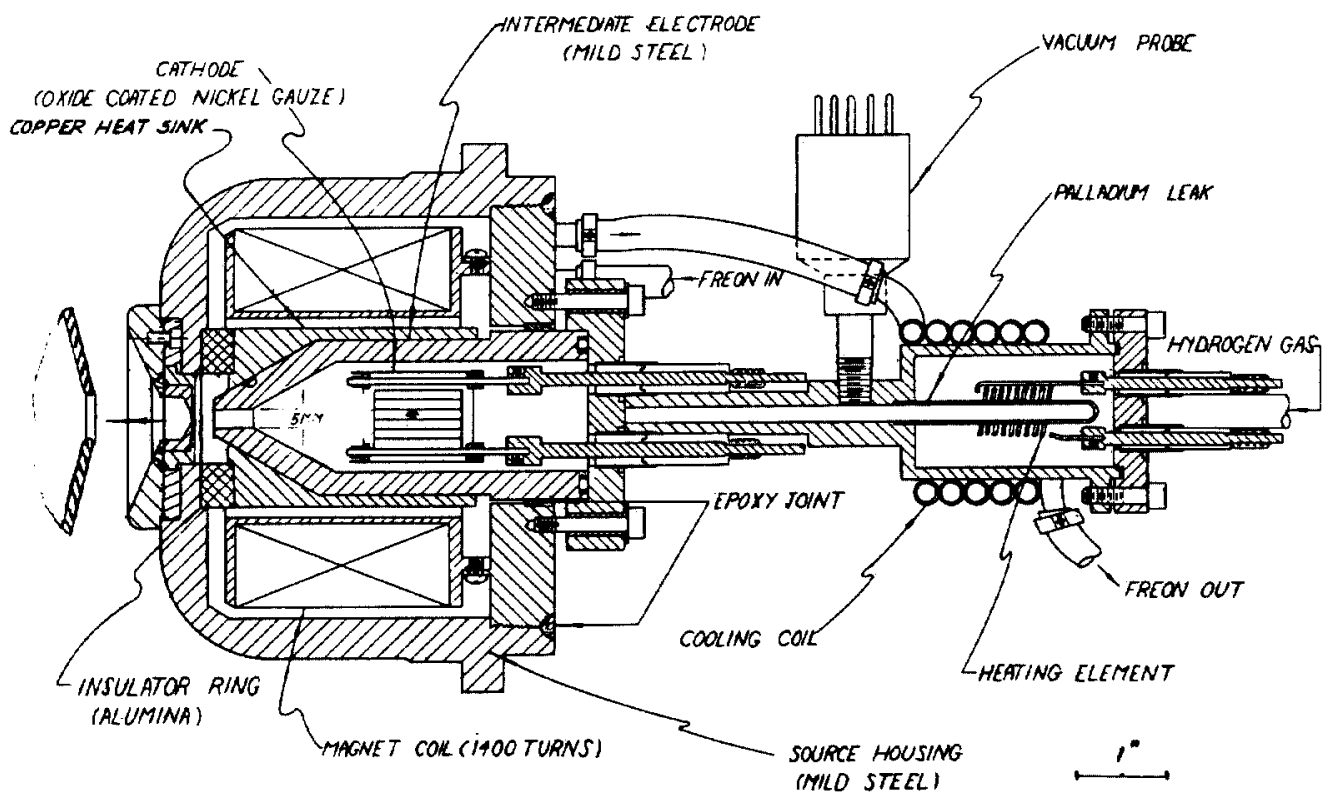

Fig. 7. BNL Duoplasmatron Ion Source. 


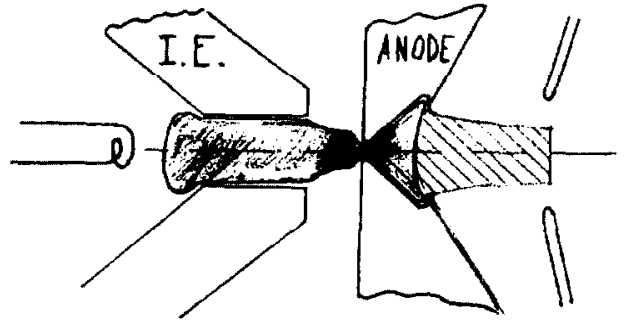

Fig. 8. Coned Cup Extraction.

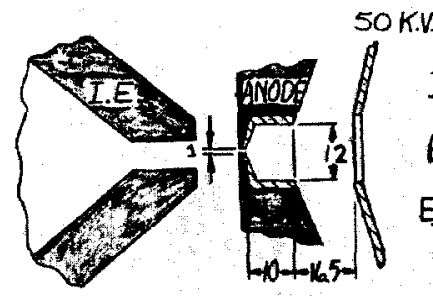

$I=100 \mathrm{MA}$

$E=.062 \mathrm{CM} \cdot M R A D$

$E_{\text {THEOR }}=.020 \mathrm{CM} \cdot \mathrm{MRAD}$
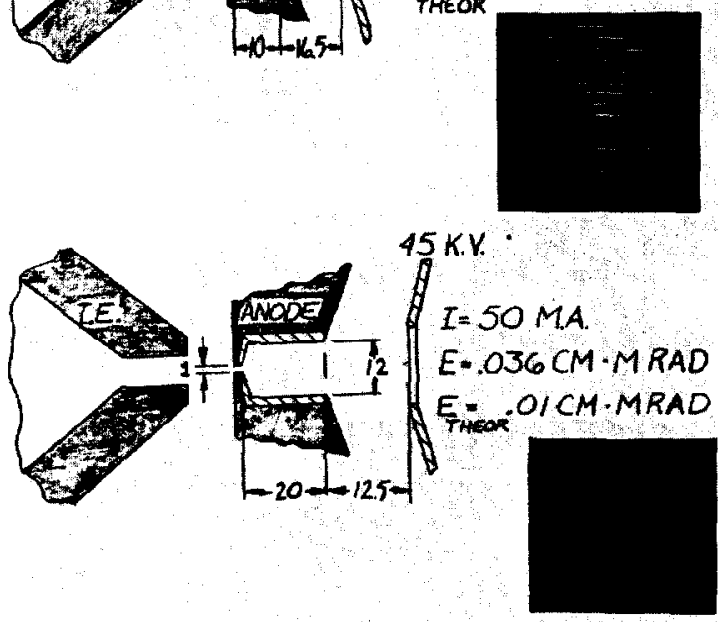

Fig. 9. Comparison of Two Non-Aberrant Nomalized Emittances.

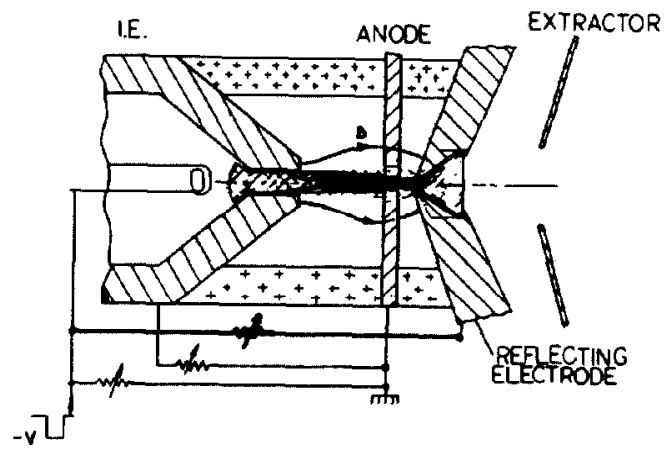

Fig. 10. Modified Duoplasmatron. 